\title{
Hardware-in-the-Loop Simulation for Machines based on a Multi-Rate Approach
}

\author{
Christian Scheifele Alexander Verl \\ Institute for Control Engineering of Machine Tools and Manufacturing Units, University of Stuttgart, Germany, \\ christian.scheifeledisw.uni-stuttgart.de
}

\begin{abstract}
The commissioning of the entire control system using a digital shadow of the machine offers extensive advantages in industrial control engineering for machine manufacturers and machine integrators. The growing use of a Hardware-in-the-Loop Simulation (HiLS) in the engineering process is accompanied by the steady increase in demands regarding model depth and model scope of the virtual machine. Especially in the area of material flow simulation, currently used simulation setups of HiL-Simulators reach their limits because of the limitation on a single simulation solver. This paper presents an approach on how a virtual machine could be realized based on several interlinked simulation solvers connected by a multi-rate approach to increase the model depth and model scope.
\end{abstract}

Keywords: hardware-in-the-loop simulation, co-simulation, multi-rate simulation, virtual commissioning, material flow simulation

\section{Introduction}

In the context of Industry 4.0, the use of digital methods and tools over the complete life cycle of a production system plays a vital role because of the increasing degree of complexity of modern production systems. In this context, one speaks of the 'virtual production': The seamless digital modelling of product installations and processes for experimentation purposes. In the area of industrial control engineering the virtual commissioning of machine tools presents great potential. Simulative methods are used more and more by machine manufacturers and machine integrators in the engineering process: In the course of virtual commissioning, the control system can be put into operation at an early development stage and before the real machine is available using a 'virtual machine'. With the aid of a virtual machine, the control system is tested regarding quality and performance. Furthermore, unforeseen errors are eliminated. In summary, virtual commissioning saves time and money and simplifies the engineering process. There is a need for further research in order to reach the vision of an encompassing virtual production.
Regarding the simulation setup of a virtual commissioning, several test configurations, such as Model-in-the-Loop (MiL), Software-in-the-Loop (SiL) and Hardware-in-the-Loop (HiL), can be distinguished. Especially in the context of CNC machines (CNC Computerized Numerical Control), Hardware-in-theLoop Simulation (HiLS) offers many advantages because the entire control system can be tested without any technical modifications or adaptations. In the context of CNC machines, the HiLS describes a test configuration, where the real control system is connected with a virtual machine based on a single simulation solver via the real communication periphery (Pritschow and Röck, 2004). However, in order to meet the requirements on a time-synchronous and lossless data processing in relation to the deterministic cycle time of the real control system, the machine simulation has to process the control outputs to control inputs in between the deterministic control cycle time (today $1 \mathrm{~ms}$ for CNC machine tools). These high demands on timedeterministic algorithms cause restrictions on the model depth and model scope of a virtual machine. There are reduction schemes and numerical integration techniques available, which enable an efficient computation of the simulation models in some cases. However, computation-intensive and non-deterministic algorithms in the field of structural mechanics (e.g. realtime capable finite element models, flexible multibody systems), process simulation (e.g. chip formation), 3Dkinematic simulation with collision detection and simulation of the dynamics of material flow systems can only be used if simplified and adapted simulation models can be found. In summary, the current simulation setup based on a single simulation solver reaches its limits considering the simulation of an encompassing virtual production.

This paper presents the objective of a simulator based on several interlinked simulation solvers with different real-time requirements and cycle times connected by a multi-rate approach to increase the model depth and model scope of a HiLS of machines. The feasibility is demonstrated by the example of a material flow.

This paper is organized as follows: In section II preliminary work is presented that is considered relevant to this research. Section III describes the current 
simulation setup of a HiLS in industrial control engineering and its limitations. On this basis, an expansion of the current simulation setup based on a multi-rate approach is motivated in section IV. The case study on a physics-based material flow simulation is demonstrated in section V. The paper closes with a summary and an outlook in section VI considering future work respective this research.

\section{Related Work}

Pritschow and Röck introduce in (Pritschow and Röck, 2004) a simulation setup of a HiLS for machine tools using a real CNC. The integration of the real CNCSystem in the simulation loop requires a timedeterministic simulation of the machine that runs under a real-time operating system. An example where this simulation setup reaches its limitations regarding computation-intensive and non-deterministic simulation models is a material flow simulation using a physicsbased simulation approach:

Physics-based material flow models are predestined for the simulation of the material flow dynamics of a virtual production. Zäh, Lacour and Spitzweg propose in (Zäh et al, 2008) a five step modelling process based on the CAD model that yields in a physical and kinematical model of a material flow system.

Few approaches address the integration of a physicsbased material flow model into a time-deterministic virtual machine (Hoher et al, 2011; Hoher and Verl, 2012; Neher and Lechler, 2015). However, Hoher and Verl demonstrate in (Hoher and Verl, 2012) that a physics-based simulation approach is only possible with a small number of moving objects (70 dynamic objects with a simplified modeling already require up to $1,5 \mathrm{~ms}$ ) within the described simulation setup of a HiLS.

In order to avoid these limitations, this paper presents the objective of an expansion of the simulation setup introduced in (Pritschow and Röck, 2004) by a multirate approach to increase the model depth and model scope. In the field of multi-rate simulation, extensive mathematical research is available (Gear and Wells, 1984) (Muttay-Smith, 1984). However, there is no solution present, which shows such a simulation setup in the field of machines and industrial control engineering. A solution needs to be found, which focus the specific scientific questions regarding the integration of a real control system in the simulation loop (HiLS), where the simulator consists of several interlinked simulation solvers.

\section{HILS of Machine Tools and its Limitations}

\subsection{Real-Time Requirements}

In a real-time simulation, the simulation time synchronizes with the real time. In the field of industrial control engineering, two different types of real-time requirements need to be distinguished with regard to used algorithms:

1. Soft real-time requirements: The simulation usually calculates simulation results in a timely manner. Significant deviations are rare but possible. Windows-based real-time simulation cores usually obey soft real-time requirements.

2. Hard real-time requirements: The simulation always calculates simulation results in a timely manner. To achieve equidistant time intervals a real-time operating system is required.

\subsection{Simulation Setup for a HiLS of Machine Tools}

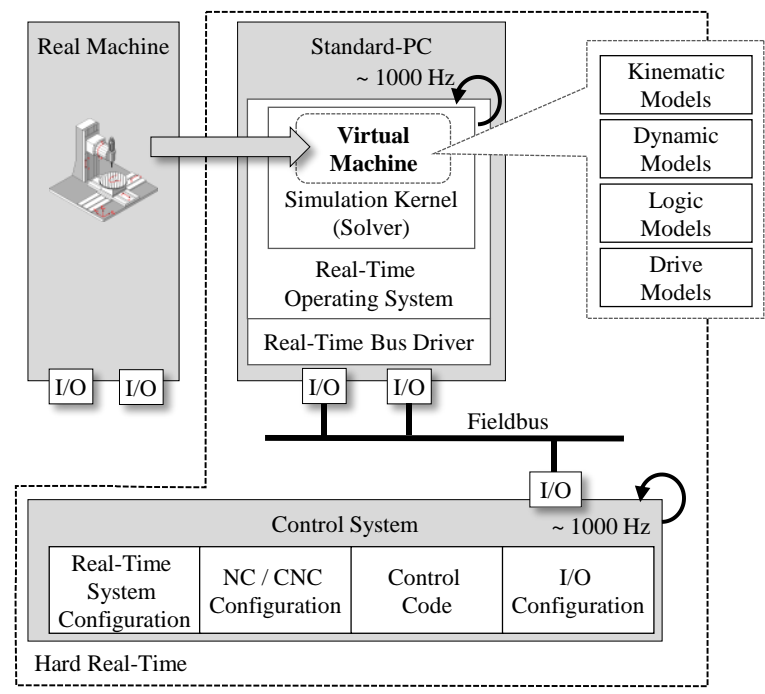

Figure 1. Simulation setup for a HiLS of machine tools using real CNC.

The decisive advantage of a HiLS of machine tools, as depicted in Figure 1, is the commissioning of an unmodified and entire control system. From the point of view of the real control system, there shell be no difference between the real and the simulated machine. To meet the requirements on a time-synchronous and lossless data processing in relation to the cycle time of the control, the simulation solver runs as real-time task on the real-time operating system (Pritschow and Röck, 2004). This ensures that time sensitive (fast changing $\mathrm{I} / \mathrm{Os}$ ) and time synchronous events (drive amplifier) can be handled by the simulator. Hence, the requirements on the time-deterministic simulation models are (Pritschow and Röck, 2004):

- A time-deterministic kernel of the underlying operating system to run the simulation solver

- The algorithms of the simulation models for the machine simulation must be time-deterministic

- Simulation cycle time $1 \mathrm{~ms}$ (same as control system cycle time)

The simulated machine consists of multiple behavior models. Starting with the I/O signals on the fieldbus, 
behavior models are required to simulate the single bus devices, such as a drive amplifier. Regarding the behavior model of a drive amplifier, it is important to react to every bit change inside the control word with correct status word. Downstream of the simulated bus devices, additional models such as kinematic, logic or dynamic models of the machine are needed to generate a realistic observation.

\subsection{Limitations of the Simulation Setup}

Following limitations can be determined:

1. Single simulation solver: The described simulation setup of a HiLS uses a single simulation solver running as real-time task on a real-time operating system. The setup applies an uniform cycle time (derived from the control system cycle time) and hard real-time requirements (simulation results must be guaranteed before the next control step starts) to all parts of the model. These two properties limit the model depth and model scope of the virtual machine, because of desired timedeterministic and performant algorithms and weak exploitation of available processing power.

2. Parallelization: Multi-core processors with an increasing number of cores as well as developments in the field of GPGPU (GPGPU - general-purpose computing on graphics processing units) are emerging since the beginning of this century offering a continuously increasing performance for simulation applications on standard PCs. A distribution of real-time tasks across different cores (Multi-core) requires a division of the simulation solver into functional units. An increase in computing performance by harnessing the power of the GPU (GPU - graphics processing unit) requires a coupling of a soft real-time simulation solver under Windows because GPGPU is currently not possible under real-time operating systems.

3. Available simulations cores: Simulation approaches of various simulation disciplines need to be combined to simulate the overall behavior of a machine or production. In the meantime, highly specialized real-time simulation cores for various simulation disciplines were developed. These simulation cores are mostly Windows-based and therefore impossible to run under a real-time operating system. This is a further argument for coupling Windows-based simulation cores.

The following amendments to the simulation setup are required to achieve an increasing model depth as well as an increasing model scope (see Figure 2):

- Splitting up of the single simulation solver into functional units: several interlinked simulation solvers with various cycle times
- Different real-time requirements to the simulation solvers: Enhancement of the simulation setup by soft real-time simulation solvers

- Usage of multi-rate and multi-step methods

- Integration of available highly specialized simulation cores

- Parallelization of simulation tasks: Multi-core and GPGPU support

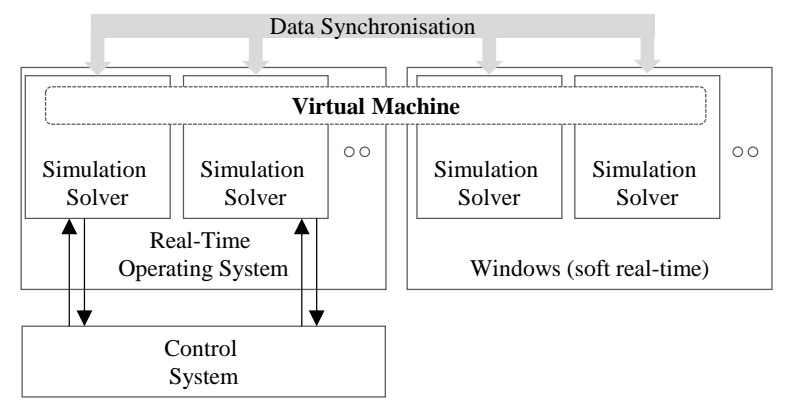

Figure 2. Multiple simulation cores.

\section{Multi-Rate Simulation Approach for Machines}

\subsection{Multi-Rate Simulation Techniques}

By splitting up of a single simulation solver into functional units with different cycle times and different real-time requirements, a synchronisation strategy is required. In the context of differential-equation models, few approaches address 'multi-rate' techniques, e.g. (Gear and Wells, 1984; Muttay-Smith, 1984).

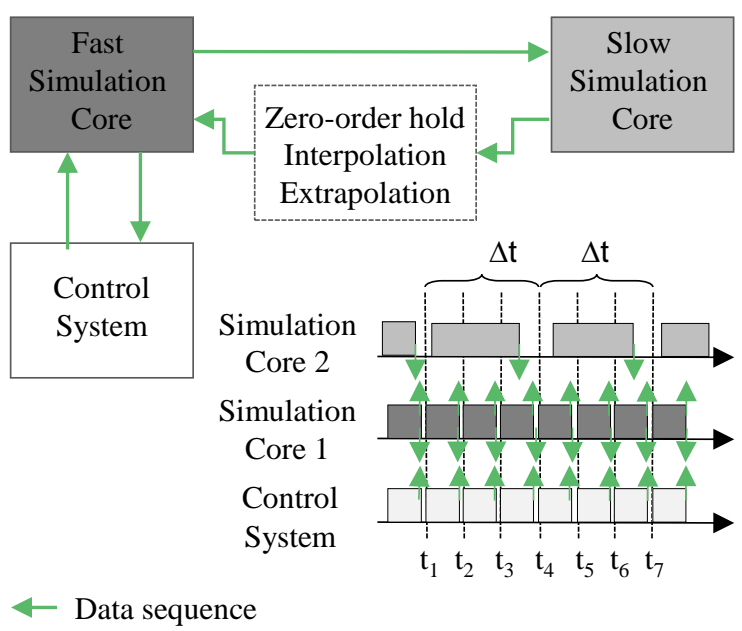

Figure 3. Multi-rate simulation.

Multi-rate methods for converting slow data sequence outputs from a slow functional unit into fast data sequence inputs for a fast functional unit can be divided into three groups, see Figure 3:

- Zero-order hold: Holding the last output $y$ from the slow functional unit as input $u$ from the fast functional unit until the output $y$ is updated $(0 \leq$ $a \leq 1)$ : 


$$
u_{n+a}=y_{n}
$$

- Interpolation algorithms: Calculation of new inputs $u$ within the range of a discrete set of known outputs $y$ e.g. first-order interpolation $(0 \leq a \leq 1)$ :

$$
u_{n+a}=y_{n}+a\left(y_{n+1}-y_{n}\right)
$$

- Extrapolation algorithms: Estimating the value $u$ from the values $y$ computed beforehand e.g. firstorder extrapolation $(0 \leq a \leq 1)$ :

$$
u_{n+a}=y_{n}+a\left(y_{n}-y_{n-1}\right)
$$

\subsection{Multi-Rate Simulation Techniques for Virtual Machines}

To transfer these techniques to a HiLS of machines, an application specific consideration is required:

1. Coupling of Soft-Real-Time-Cores: The coupling of simulation cores in soft real-time is of great interest. Soft real-time algorithms can't guarantee the calculation of simulation results if the cycle time is choosen too low. An appropriate choice of the cycle time is necessary. Therefore, the worst case need to be calculated in advance. Furthermore, a simulation step of a simulation core on Windows has to be commanded from a task on the real-time operating system.

2. Allocation of multi-rate methods: The choice of an available multi-rate method depends on the behavior model as well as on the characteristic of a signal. Furthermore, multiscale modeling can be considered: In a fast simulation core, a simplified and performant simulation approach is realized which is guided by a slow simulation core based on a precise simulation approach.

3. Accuracy and stability: Whether a behavior model requires the same cycle time as the control system is depending on the characteristic of the linked $\mathrm{I} / \mathrm{O}$ signals of the control system. The effects of signal jumps as well as inaccuracies because of the multirate method has to be considered. Furthermore, control commands could have the demand on immediate processing by the simulation.

4. Look-ahead simulation: For the use of interpolation multi-rate methods, it might be necessary to parallel computing of the same simulation core with different cycle times. Furthermore, simulations faster than real-time can also be considered. As long as there is no control command, these calculations can be correct.

5. Communication between cores: A performant data exchange between the simulation cores is very important. Communication in-between the realtime operating system as well as between Windows and the real-time operating system has to be taken into account.

\section{Case Study on a Physics-Based Material Flow Simulation}

The high demands on time-deterministic algorithms is especially within the context of material flow simulation a major problem. In modern production systems, several conveyor systems combine individual machines to a material flow system. The real control system, in this case the individual machine controls as well as the superordinated production control, can be connected with a virtual production in a HiLS if a material-flow model is available. For example, looking at machines from packaging or beverage industry, the state of the control, the plant layout and operational throughput are directly related to the physical and geometric properties of the material flow. The material flow consists of a high

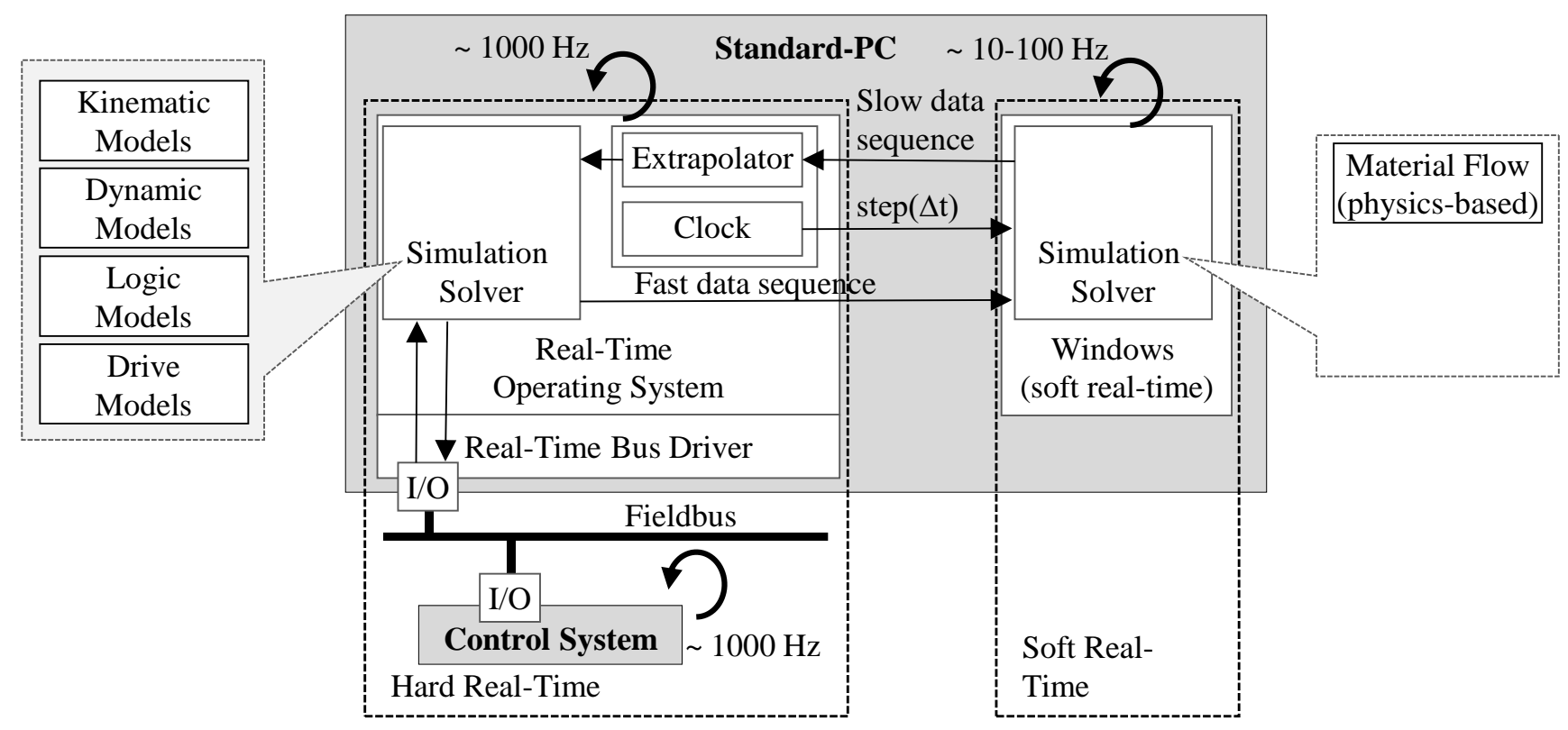

Figure 4. Simulation setup for HiLS based on a multi-rate approach of machines embedded in a material flow system. 
number of moving objects (> 1000), which interact with each other over collisions. A behavior model for the dynamics of the material flow has to be found to simulate the exact process sequences and to reproduce a realistic interaction with the control system via fieldbus I/Os.

A physics-based simulation approach calculates the object motion on runtime of a simulation based on the laws of classical mechanics (rigid-body simulation). Physical (e.g. mass, coefficient of friction) and geometric (collision shapes) properties of the simulation objects as well as the object arrangement (positions and orientations) and simulation scene properties (e.g. force of gravitation) are required for modelling the initial configuration of a simulation scene. Full-grown simulation cores, called physics engines, for the simulation of physical systems are available. These physics engines provide rigid body dynamics including collision detection and can be used for a material flow simulation. However, these physics engines are not based on time-deterministic algorithms and meet only soft real-time requirements. Thus, the physics engines cannot be executed on a real-time operating system for a high number of moving objects. For coupling the physics engine, running on Windows, a suitable multi-rate method has to be found. Furthermore, the simulation core on Windows has to be commanded from a task on the real-time operating system. Figure 4 shows the simulation setup.

Regarding a conveyor belt with 245 moving cylinders (see Figure 5), the computation time of a physics-based simulation for a $40 \mathrm{~ms}$ time step is about $18-40 \mathrm{~ms}$ (see Figure 6). Thus, an appropriate method has to be found, which provides the inputs for the time-deterministic simulation solver $(\sim 1 \mathrm{~ms})$.

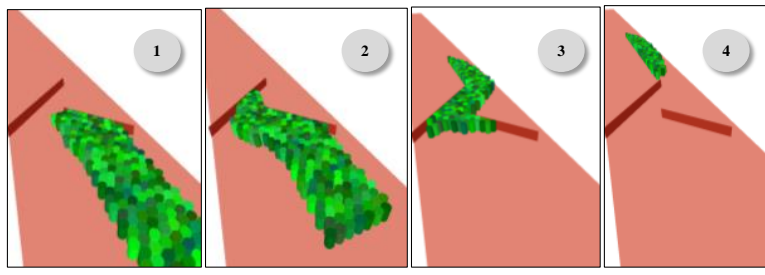

Figure 5. Material flow scene: Conveyor belt with 245 moving cylinders.

One option would be to use a zero-order hold multirate model. As described, this method would hold the last position vector from the slow simulation core as input for the fast simulation core until the position vector is updated.

A better option would be to use a multiscale modeling approach. A pure kinematic simulation, which lead the objects on predefined trajectories, is possible under hard real-time conditions. A kinematic simulation (in this case only translational movements) calculates in each simulation step the next position vector $r_{n+1}$ of an object $\underline{\mathrm{r}}_{\mathrm{i}+1}$ by the current position $\underline{r}_{n}$, the current velocity vector $v_{n}$ and the simulation time step $\Delta t$ :

$$
\underline{r}_{n+1}=\underline{r}_{n}+\underline{v}_{n} \cdot \Delta t \quad \underline{\mathrm{r}}_{\mathrm{i}+1}=\underline{\mathrm{r}}_{\mathrm{i}}+\underline{\mathrm{v}}_{\mathrm{i}} \cdot \Delta \mathrm{t}
$$

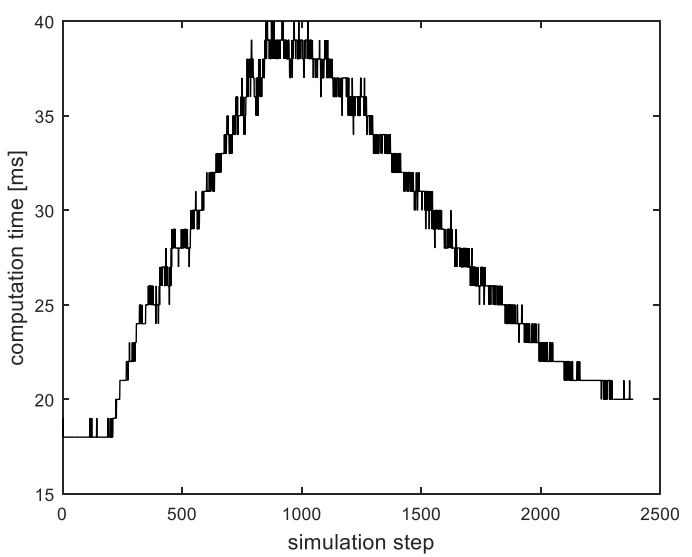

Figure 6. Computation time of the physics-based simulation.

On this basis, a multi-rate method can be developed: At $\mathrm{n}=0$, the physics-based simulation model, which is running on the slow simulation core, sends the current precise position vector $\underline{r}_{p}$ and velocity vector $\underline{v}_{p}$ of the object. The multi-rate method now updates the current position vector (2) and uses the velocity vector to generate new position vectors (3) until a new data set arrives from the slow simulation core:

$$
\begin{gathered}
\underline{r}_{n+0}=\underline{r}_{p} \\
\underline{r}_{n+a}=\underline{r}_{n}+\underline{v}_{n} \cdot \Delta t
\end{gathered}
$$
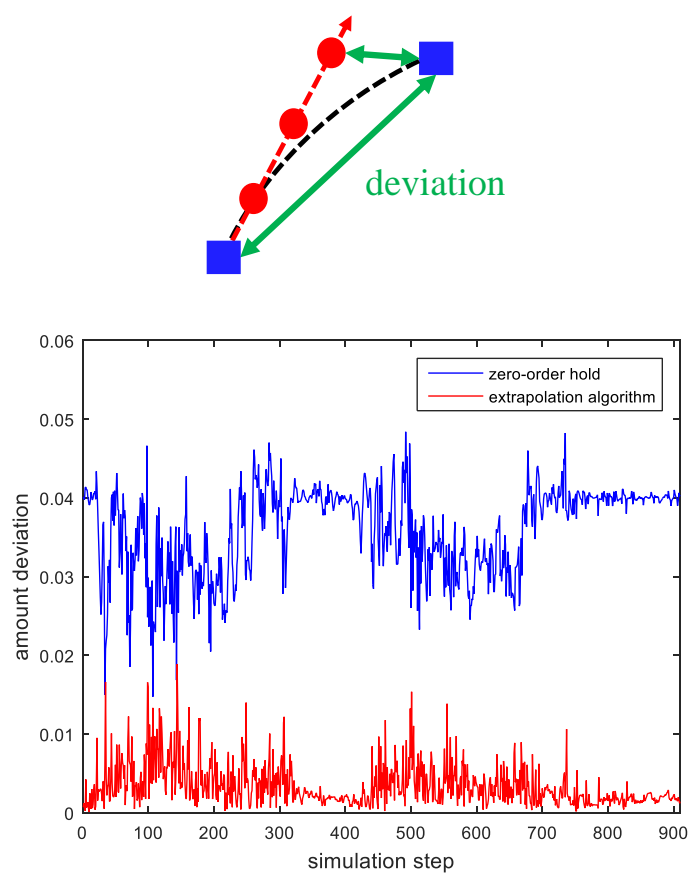

Figure 7. Amount deviation with extrapolation algorithm.

In this example, the soft real-time simulation solver with the physics-based simulation runs with a simulation cycle time of $40 \mathrm{~ms}$ and the hard real-time simulation solver runs with a time-deterministic cycle time of $1 \mathrm{~ms}$. Compared with a zero-order hold multi- 
scale method, the usage of the multiscale modeling approach enhances the model accuracy, see Figure 7. It can be seen that the accuracy of a coupled physics-based simulation is sufficient for the requirements of a HiLS of machines.

The case study is implemented in a real-time environment using Beckhoff TwinCAT real-time extension for Microsoft Windows 7 64bit. For the physics-based simulation, the physics engine NVIDIA PhysX SDK 3.3.3 and for the virtual machine the simulation tool ISG-virtuos is used.

\section{Summary and Outlook}

The requirements regarding model depth and model scope of the virtual machine in a HiLS are continually on the rise. The currently used simulation setup reaches its limits because of the limitation on a single simulation solver. To master these demands the division of the single simulation solver into functional units based on multi-rate methods should be considered, so that multicore and GPGPU technologies as well as a wider range of simulation algorithms with different real-time requirements and cycle times can be used to achieve the given objective of a virtual production. Therefore a precise observation is necessary, to keep meeting the requirements on a time-synchronous and lossless data processing in relation to the cycle time of the control system.

Out of this context, this paper presents the objective of a simulator based on several interlinked simulation solvers with different real-time requirements and cycle times connected by a multi-rate approach to increase the model depth and model scope of a HiLS of machines. The feasibility is demonstrated by the example of a material flow simulation.

The objective and future work is an in depth analysis of the described approach and the analysis of the interaction with an industrial control system.

\section{References}

C. W. Gear and D. R. Wells: "Multirate linear multistep methods," BIT Numerical Mathematics, 24(4):484.502, 1984. doi: 10.1007/BF01934907

S. Hoher, P. Schindler, S. Göttlich, V. Schlepper and S. Röck. System Dynamic Models and Real-time Simulation of Complex Material Flow Systems. 4th International Conference on Changeable, Agile, Reconfigurable and Virtual Production (CARV11), 316-321, Springer, 2011. doi: 10.1007/978-3-642-23860-4_52

S. Hoher and A. Verl. A Multi Simulator Approach of Material Flow Systems for Virtual Commissioning. SPSIPCDRIVES 2012 Tagungsband, G. Frey, W. Schumacher, A. Verl, 387-396, VDE, 2012.

D. J. Murray-Smith. Modelling and simulation of integrated systems in engineering: issues of methodology, quality, testing and application. Elsevier, 2012.
P. Neher and A. Lechler. Using game physics engines for hardware-in-the-loop material flow simulations: benefits, requirements and experiences. Advanced Intelligent Mechatronics (AIM), 2015 IEEE International Conference on. IEEE, 2015. doi: 10.1109/AIM.2015.7222670

G. Pritschow and S. Röck. Hardware in the Loop Simulation of Machine Tools. CIRP Annals-Manufacturing Technology, 53(1):295-298, 2004. doi:10.1016/S00078506(07)60701-X

M. F. Zäh, F. Lacour and M. Spitzweg. Application of a physical model for the simulation of the material flow of a manufacturing plant. IT - Information Technology, 50(3):192-198, 2008. 